\title{
The potential and value of objective eye tracking in the ophthalmology clinic
}

\author{
Rosie Clark ${ }^{1} \cdot$ James Blundell $\oplus^{2} \cdot$ Matt J. Dunn $\oplus^{3} \cdot$ Jonathan T. Erichsen ${ }^{3} \cdot$ Mario E. Giardini $\oplus^{4} \cdot$ Irene Gottlob $^{5}$.

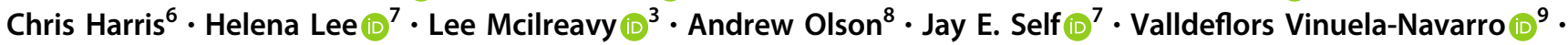 \\ Jonathan Waddington $^{10} \cdot$ J. Margaret Woodhouse $^{3} \cdot$ lain D. Gilchrist ${ }^{11} \cdot$ Cathy Williams $^{1}$
}

Received: 22 January 2019 / Accepted: 23 January 2019 / Published online: 8 April 2019

(c) The Royal College of Ophthalmologists 2019

Numerous research studies have demonstrated the scope and value of eye movement recording (EMR). There is now potential for EMR to be helpful in a range of clinical contexts and it could be developed as a routine part of the repertoire of clinical investigations offered by the NHS, at least in tertiary centres. We highlight potential uses and challenges below, as a prelude to further development and debate.

\section{Diagnosis}

EMR in patients with nystagmus is already increasingly used clinically and provides the only method for identifying the exact waveform [1,2]. A classic example is identifying the characteristic accelerating waveform of infantile nystagmus syndrome (INS), which obviates the need for urgent investigations of newly diagnosed nystagmus, saving the patients and the NHS time and money. EMR may also indicate the cause of an abnormal head posture (AHP) and identify the best option for treatment. For example, an AHP may be adopted to use a null point in subclinical, previously

Cathy Williams

Cathy.Williams@bristol.ac.uk

1 Population Health Sciences, Bristol Medical School, Bristol University, Bristol, UK

2 Institute of Future Transport and Cities, Coventry University, Coventry, UK

3 School of Optometry and Vision Sciences, Cardiff University, Cardiff, UK

4 Department of Biomedical Engineering, University of Strathclyde, Glasgow, UK

5 Department of Neuroscience, Psychology \& Behaviour, University of Leicester, Leicester, UK undiagnosed INS or to put an eye into adduction if the patient has latent nystagmus.

However, EMR can help in the management of patients other than those with nystagmus. Examples include:

In Parkinson's disease, EMRs of saccades help differentiate between dementia with Lewy bodies, progressive supranuclear palsy, corticobasal degeneration and multiple system atrophy [3].

EMR will differentiate between Gaucher Disease Type 1 and Type 3 [4]. This is particularly important, as there are different treatment pathways for these patient groups. Abnormal EMR metrics have also been reported in children with three rare metabolic diseases: Tyrosinemia III, Niemann Pick C, and Morquio syndrome [5, 6] — potentially allowing treatment to be started earlier in the disease process [6].

In psychiatry, EMR performance on the antisaccade task is affected (see: [7]) and EMR metrics have been used to classify cases of schizophrenia vs controls with 87 98\% accuracy [8], which again may allow earlier and more accurate diagnosis, with earlier treatment and support.
6 School of Psychology Plymouth University, UK \& Royal Eye Infirmary, Derriford Hospital, Plymouth, UK

7 Clinical and Experimental Sciences, University of Southampton, Southampton, UK

8 School of Psychology, University of Birmingham, Birmingham, UK

9 Ophthalmic Research Group, Life and Health Sciences, Aston University, Aston, UK

10 Research and Development, WESC Foundation, Exeter, UK

11 School of Psychological Science, Bristol University, Bristol, UK

. 


\section{Screening of at-risk individuals}

There is a growing body of evidence that EMR may be useful in the screening of individuals at risk of disorders, including Huntington's [9], Alzheimer's [10, 11] and Parkinson's [12] diseases.

\section{Monitoring of disease progression and of response to treatment}

The EMR abnormalities in Niemann Pick C, including curved saccades, increase in magnitude with disease severity suggesting that these measures would also be useful in monitoring disease progression. Also, in Parkinson's disease, the extent of EMR abnormalities is related to disease progression [13] and responsiveness to treatment [14].

Although these results are encouraging, it is likely that EMR alone will only rarely, if ever, be used as the only diagnostic criterion. However, diagnostic pathways that include EMR alongside, for example magnetic resonance imaging [15], are likely to be shorter and more accurate. While the individual conditions may be rare, such as the metabolic disorders, there are a much larger number of patients who present with early or non-specific difficulties in whom treatable metabolic or neurological disease needs to be ruled out, and therefore, specialist services that care for many patient groups may benefit from access to reliable EMR within the NHS.

The objective and quantitative measurement of eye movements has a long history dating back to the early 20th Century [16]. Early methods were uncomfortable and invasive, and analysis of the resulting data was timeconsuming. However, the advent of both powerful personal computing and fast video-based recording systems has led to a step-change in the past 15 years in this technology. EMR has become standard in a wide range of settings, including consumer research, human-computer interaction and virtual reality. Alongside this, work on both the neurophysiology of eye movement control [17] and the detailed study of human eye movement behaviour [18] means that we can map this visual-motor behaviour onto the patterns of activity across a well-studied and extensive brain network.

Routine recording of eye movements in a specialist clinical setting is now therefore technically feasible and would provide a sensitive, quantitative and objective method to aid diagnosis and management for a range of patients. However, despite this potential benefit as a clinical tool, there are considerable challenges associated with both introducing eye tracking into clinical practice and making it cost-effective. We are still some way from having eye- tracking hardware that is able to successfully record the eye movements of every patient, whatever their age and level of ability. We need a common suite of behavioural assays that are agreed upon by the wider community, with normative data [19].

We would need to identify which groups of staff would carry out the assessments, what training they would need and how and by whom the resulting data should be reported. One model is to develop inbuilt test paradigms and proforma reports that include normative data, to limit the expertise required by the individual setting up the test and make EMR accessible to a range of users. However, this highlights the important issue of expertise in interpreting clinical eye movements. EMR may be used to look for very specific abnormalities in an individual patient and a targeted approach (as opposed to a general battery of tests) may be important for efficiency and to address the key clinical question for that patient, especially for children. The choice between a targeted or comprehensive approach requires both technical ability and specific expertise. Training is required, but experience is also important (as any clinician knows). Currently, there is no training offered and no recognised training pathway. One possible route is to set up training for eye movement clinical scientists that could eventually become registrable with the Health and Care Professions Council (HCPC). As an example, one of us (C. H.) is registered with the HCPC as a 'Clinical Scientist' (which is a protected title) with designated expertise in eye movements (the only one we are aware of). This avenue could be explored as a way forward to formalise (and regulate) clinical oculomotor expertise.

EMR is already widely used in advertising, the aviation industry, rehabilitation services, computer gaming and virtual reality equipment. The time has come to explore how best to deploy this technology to the benefit of patients and the NHS.

Author contributions All authors contributed to the drafting and/or revision of this article. The manuscript was coordinated by R.C., I.D.G. and C.W.

Funding The work was supported by a grant from the UK Engineering and Physical Sciences Research Council (EP/M000885/1) to the Bristol Vision Institute, which supported R.C. and a 1-day workshop on this topic at the University of Bristol on 24 April 2018. C.W. is supported by an NIHR senior research fellowship (SRF208-015).

\section{Compliance with ethical standards}

Conflict of interest The authors declare that they have no conflict of interest.

Publisher's note: Springer Nature remains neutral with regard to jurisdictional claims in published maps and institutional affiliations. 


\section{References}

1. Papageorgiou E, McLean RJ, Gottlob I. Nystagmus in childhood. Pediatr Neonatol. 2014;55:341-51.

2. Dunn M. Clinical assessment of nystagmus. Optometry Today. 2016;56:80-85.

3. Armstrong RA. Oculo-visual dysfunction in Parkinson's disease. J Parkinsons Dis. 2015;5:715-26.

4. Harris CM, Taylor DS, Vellodi A. Ocular motor abnormalities in Gaucher disease. Neuropediatrics. 1999;30:289-93.

5. Blundell J, Frisson S, Chakrapani A, Kearney S, Vijay S, MacDonald A, et al. Markers of cognitive function in individuals with metabolic disease: Morquio syndrome and tyrosinemia type III. Cogn Neuropsychol. 2018;35:120-47.

6. Blundell J, Frisson S, Chakrapani A, Gissen P, Hendriksz C, Vijay $\mathrm{S}$, et al. Oculomotor abnormalities in children with Niemann-Pick type C. Mol Genet Metab. 2018;123:159-68.

7. Hutton SB, Ettinger U. The antisaccade task as a research tool in psychopathology: a critical review. Psychophysiology. 2006; 43:302-13.

8. Benson PJ, Beedie SA, Shephard E, Giegling I, Rujescu D, St Clair D. Simple viewing tests can detect eye movement abnormalities that distinguish schizophrenia cases from controls with exceptional accuracy. Biol Psychiatry. 2012;72:716-24.

9. Blekher TM, Yee RD, Kirkwood SC, Hake AM, Stout JC, Weaver $\mathrm{MR}$, et al. Oculomotor control in asymptomatic and recently diagnosed individuals with the genetic marker for Huntington's disease. Vision Res. 2004;44:2729-36.

10. Crawford TJ, Higham S, Renvoize T, Patel J, Dale M, Suriya A, et al. Inhibitory control of saccadic eye movements and cognitive impairment in Alzheimer's disease. Biol Psychiatry. 2005; 57:1052-60.

11. Boxer AL, Garbutt S, Seeley WW, Jafari A, Heuer HW, Mirsky J, et al. Saccade abnormalities in autopsy-confirmed frontotemporal lobar degeneration and Alzheimer disease. Arch Neurol. 2012; 69:509-17.

12. White OB, Saint-Cyr JA, Tomlinson RD, Sharpe JA. Ocular motor deficits in Parkinson's disease. II. Control of the saccadic and smooth pursuit systems. Brain. 1983;106:571-87.

13. Jankovic J. Parkinson's disease: clinical features and diagnosis. J Neurol Neurosurg Psychiatry. 2008;79:368-76.

14. Hood AJ, Amador SC, Cain AE, Briand KA, Al-Refai AH, Schiess MC, et al. Levodopa slows prosaccades and improves antisaccades: an eye movement study in Parkinson's disease. J Neurol Neurosurg Psychiatry. 2007;78:565-70.

15. Rodrigue AL, Schaeffer DJ, Pierce JE, Clementz BA, McDowell JE. Evaluating the specificity of cognitive control deficits in schizophrenia using antisaccades, functional magnetic resonance imaging, and healthy individuals with poor cognitive control. Front Psychiatry. 2018;9:107.

16. Wade NJ, Tatler BW. Origins and applications of eye movement research. In ID Gilchrist \& S Everling, editors. The Oxford handbook of eye movements. Oxford: Oxford University Press; 2011.

17. Wurtz RH. Using perturbations to identify the brain circuits underlying active vision. Philos Trans R Soc B. 2015;370:20140205.

18. Liversedge SP, Gilchrist ID, Everling S. The Oxford handbook of eye movements. Oxford: Oxford University Press; 2011.

19. Antoniades C, Ettinger U, Gaymard B, Gilchrist ID, Kristjansson A, Kennard C, et al. An internationally standardised antisaccade protocol for clinical use. Vision Res. 2013;84:1-5. 